\title{
Inhibition of lactate dehydrogenase A by microRNA-34a resensitizes colon cancer cells to 5-fluorouracil
}

\author{
XIANGYONG LI ${ }^{1}$, HAIBIN ZHAO ${ }^{2}$, XIJIAN ZHOU ${ }^{1}$ and LEI SONG ${ }^{1}$ \\ Departments of ${ }^{1}$ Hematology and Oncology, and ${ }^{2}$ Pathology, \\ The 101st Hospital of the People's Liberation Army, Wuxi, Jiangsu 214044, P.R. China
}

Received January 16, 2014; Accepted August 22, 2014

DOI: $10.3892 / \mathrm{mmr} .2014 .2726$

\begin{abstract}
Fluorouracil (5-FU) chemotherapy is widely used in the treatment of advanced colon cancer. However, the development of resistance to 5-FU is a significant obstacle to successful treatment. MicroRNA-34a (miR-34a) has been reported to be downregulated in a number of tumor types and has also been shown to act as a tumor suppressor. However, the mechanisms underlying the biological effects of miR-34a in chemoresistance remain unclear. The present study showed that the expression of miR-34a is downregulated in 5-FU-resistant colon cancer cells. In addition, 5-FU-resistant colon cancer cells exhibited upregulation of lactate dehydrogenase A (LDHA) expression and activity compared with parental cells. Furthermore, LDHA was shown to be a direct target of miR-34a. Overexpression of miR-34a reduced the expression of LDHA, probably through binding to the 3 ' untranslated region, leading to the re-sensitization of 5-FU-resistant cancer cells to 5-FU. Additionally, overexpression of LDHA rendered colon cancer cells resistant to 5-FU, suggesting that the miR-34a-induced sensitization to $5-\mathrm{FU}$ is mediated through the inhibition of LDHA. In conclusion, the current study showed that miR-34a is involved in sensitivity to 5-FU in part through its effects on LDHA expression. This indicates that miR-34a-mediated inhibition of glucose metabolism may be a therapeutic target in patients with chemoresistant colon cancer.
\end{abstract}

\section{Introduction}

MicroRNAs (miRNAs) are short noncoding RNAs, 18 to 25 nucleotides in length, which regulate gene expression $(1,2)$. They are essential to a number of biological processes, such as embryogenesis, development, cell growth, cell differentiation and cell death $(2,3)$. Recent studies have shown that miRNAs

Correspondence to: Dr Xiangyong Li, Department of Hematology and Oncology, The 101st Hospital of the People's Liberation Army, 101 North Xiangyuan Road, Wuxi, Jiangsu 214044, P.R. China

E-mail: lixiangyong101@163.com

Key words: 5-fluorouracil, microRNA-34a, lactate dehydrogenase A, chemoresistance, colon cancer regulate a large number of oncogenes and tumor suppressor genes $(4,5)$. Therefore, understanding the downstream targets of a number of miRNAs is important in diagnostic therapeutic applications for human cancer.

5-Fluorouracil (5-FU) is a commonly-used chemotherapeutic agent that is effective for treating a range of malignant tumors (6). 5-FU is converted to fluorodeoxyuridine monophosphate, which forms a stable complex with thymidylate synthase and thus inhibits deoxythymidine monophosphate production $(7,8)$. However, a primary cause of treatment failure in advanced colon cancer is the development of chemoresistance to 5-FU. Currently, the overall response rate for advanced colorectal cancer to 5-FU alone remains at only 10-15\% (9), and the combination of 5-FU with other antitumor drugs has improved the response rates to $40-50 \%$ (10). Therefore, a novel therapeutic strategy is required to target cellular signaling molecules in order to overcome chemoresistance to colorectal cancer treatments.

The Warburg effect describes the phenomenon by which cancer cells exhibit dysregulated aerobic glycolysis regardless of their oxygen status. It has been intensively investigated and is recognized as one of the characteristic hallmarks of cancer cells in the current understanding of cancer cell metabolism $(11,12)$. Furthermore, cancer cells were found to require high levels of glucose and to be sensitive to changes in glucose concentration $(13,14)$. Lactate dehydrogenase A (LDHA) is one of the primary isoforms of $\mathrm{LDH}$ expressed in cancer tissue. It controls the conversion of pyruvate to lactate during the cellular glycolytic process. It has been shown that LDHA is involved in cancer cell glycolysis and growth, and tumor maintenance (15). Furthermore, it has been reported that LDH activity may be a reliable prognostic marker in certain types of cancer (16).

The present study investigated the effect of miR-34a-mediated glucose metabolism inhibition, via targeting of the $3^{\prime}$ untranslated region (UTR) region of LDHA, on the mechanism of 5-FU resistance in colon cancer cells.

\section{Materials and methods}

Cell lines and culture. Cells from the DLD-1 human colon cancer cell line were obtained from the American Type Culture Collection (Manassas, VA, USA). Cells were cultured in RPMI-1640 (Sigma-Aldrich, Hong Kong, China) 
supplemented with $10 \%$ fetal bovine serum (Sigma-Aldrich) and $1 \mathrm{X}$ penicillin-streptomycin-glutamine (10378-016; Invitrogen Life Technologies, Carlsbad, CA, USA) at $37^{\circ} \mathrm{C}$ in a humidified incubator with $95 \%$ air and $5 \% \mathrm{CO}_{2}$.

Generation of the 5-FU-resistant cell line. The 5-FU-resistant cell line from DLD-1 human colon cancer cells was generated as described previously (17). Briefly, DLD-1 cells were treated with gradually increasing concentrations of 5-FU in regular cell culture conditions in order to select resistant cells. Following successive treatments for a duration of up to three months, resistant cell clones were pooled and used for all subsequent experiments in this study. Resistant cells were treated with 5-FU each month for elimination of the cells which may have regained sensitivity to 5-FU.

Antibodies and reagents. Rabbit polyclonal LDHA and $\beta$-actin antibodies were obtained from Cell Signaling Technology, Inc., (Danvers, MA, USA; \#2012 and \#4967, respectively) and 5-FU antibodies were obtained from Sigma-Aldrich.

Western blot analysis. Whole cells were lysed in 1X SDS sample buffer, resolved by electrophoresis using SDS-PAGE and transferred to nitrocellulose membranes (Bio-Rad Laboratories, Hercules, CA, USA). The membranes were probed with primary antibodies overnight and incubated with horseradish peroxidase-conjugated polyclonal goat anti-rabbit immunoglobulin G secondary antibodies (Thermo Fisher Scientific, Waltham, MA, USA) for $3 \mathrm{~h}$, prior to detection using a Super Signal Enhanced Chemiluminescence kit (Pierce Biotechnology, Inc., Rockford, IL, USA). For sequential blotting, membranes were stripped with Stripping Buffer (Pierce Biotechnology, Inc.) and re-probed with other primary antibodies.

Cell viability assay. A colorimetric assay using the tetrazolium salt, MTT (EMD Millipore, Billerica, MA, USA), was used to assess the cytotoxicity of the anticancer agent, 5-FU. Single-cell suspensions were prepared and cell density was measured.MTT assays were performed according to the manufacturer's instructions. Briefly, an equal number of cells were added into each well with culture medium containing normal concentrations of either 5-FU or phosphate-buffered saline for the untreated control. Following four days of culture, $0.1 \mathrm{mg}$ MTT was added to each well and was incubated at $37^{\circ} \mathrm{C}$ for an additional $4 \mathrm{~h}$. Plates were centrifuged at $450 \mathrm{x} \mathrm{g}$ for $5 \mathrm{~min}$ at room temperature, and the medium was discarded. Dimethyl sulfoxide $(0.15 \mathrm{ml})$ was added to each well to solubilize the crystals, and the plates were immediately read at $540 \mathrm{~nm}$ on a scanning multiwell spectrometer (Bio-Tek instruments, Inc., Burlington, VT, USA). All experiments were performed three times.

Pre-miRNA transfection. miRNA precursors (pre-miRNAs) and pre-miR negative controls were purchased from Applied Biosystems Life Technologies (Foster City, CA, USA). Lipofectamine $^{\circledR} 2000$ (Invitrogen Life Technologies) was used for the transfection of pre-miRNAs. At $48 \mathrm{~h}$ following transfection, the expression of miR-34a was detected by reverse transcription-quantitative polymerase chain reaction
(RT-qPCR), and the expression of LDHA, a target of miR-34a, was measured using western blotting.

Plasmid DNA transfection. Transfection was performed using the Lipofectamine 2000 Transfection reagent (Invitrogen Life Technologies) according to the manufacturer's instructions. Overexpression vectors containing wild type LDHA (Myc-DDK-tagged) were purchased from OriGene Technologies, Inc. (Rockville, MD, USA). At 48 h following transfection, cells were collected or whole-cell lysates were prepared for further analysis.

$R T-q P C R$. RNA was extracted from cancer cells using TRIzol reagent (Invitrogen Life Technologies). cDNA synthesis was performed using a SuperScript First-Standard Synthesis system for RT-qPCR (Invitrogen Life Technologies) according to the manufacturer's instructions. qPCR analyses were performed using Assay-on-Demand primers and the TaqMan Universal PCR Master Mix reagent (Applied Biosystems Life Technologies). Samples were analyzed using an ABI Prism 7700 Sequence Detection system (Applied Biosystems Life Technologies). The following primers were used: LDHA, forward 5'-TGGAGTGGAATGAATGTTGC-3', reverse 5'-ATAGCCCAGGATGTGTAGCC-3'; and $\beta$-actin, forward 5'-AGGCACCAGGGCGTGAT-3', and reverse 5'-GCCCACATAGGAATCCTTCTGAC-3'. LDHA expression levels were normalized to those of $\beta$-actin. For miRNA expression analysis, RT-qPCR was conducted using the TaqMan microRNA reverse transcription kit (Applied Biosystems) and TaqMan microRNA assays kit (Applied Biosystems) according the manufacturer's instructions. All reactions were performed in triplicate. Human U6 served as an internal control. The relative quantities of mRNA were calculated using the comparative $\mathrm{C}_{\mathrm{T}}$ method (17). Experiments were performed three times.

Lactate production assay. Lactate production was detected using a Lactate assay kit (BioVision, Inc., Milpitas, CA, USA). Results were normalized to the quantity of total protein in the control cells.

LDH activity assay. Total LDH activity in cell lysates was examined using the LDH cytotoxicity assay kit (BioVision, Inc.) according to the manufacturer's instructions. Briefly, $2 \times 10^{5}$ cells were seeded in a 24 -well plate one day prior to the assay and all samples were analyzed in triplicate. Cells were collected and washed, and protein was extracted in order to measure LDH activity. Results were normalized to the quantity of total protein in the control cells.

Luciferase assays. The DLD-1 cells were plated at $5 \times 10^{4}$ cells/well in 24 -well plates. The following day, the cells were co-transfected with luciferase reporter plasmids (pMIR-REPORT ${ }^{\mathrm{TM}}$ miRNA Expression Reporter Vector System; Invitrogen Life Technologies; AM5795); with wild type 3'-UTR or mutant 3'-UTR of LDHA, and pre-miR-34a or pre-miR-negative (control miR; Applied Biosystems), using Lipofectamine ${ }^{\circledR} 2000$ reagent (Invitrogen Life Technologies; 11668019). Forty-eight hours post-transfection the cells were harvested and lysed using passive lysis buffer 
A

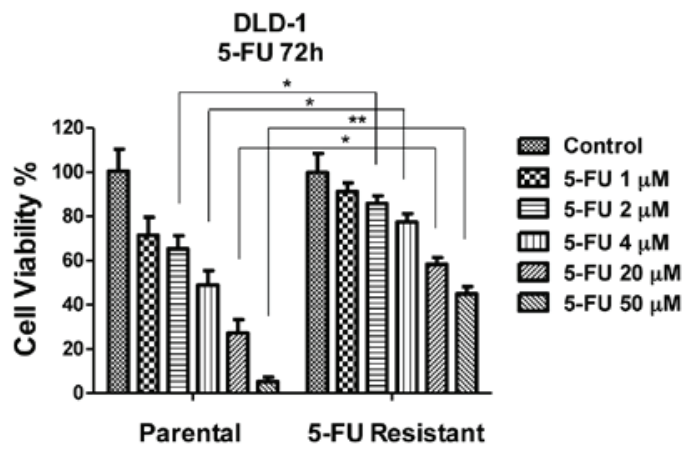

B

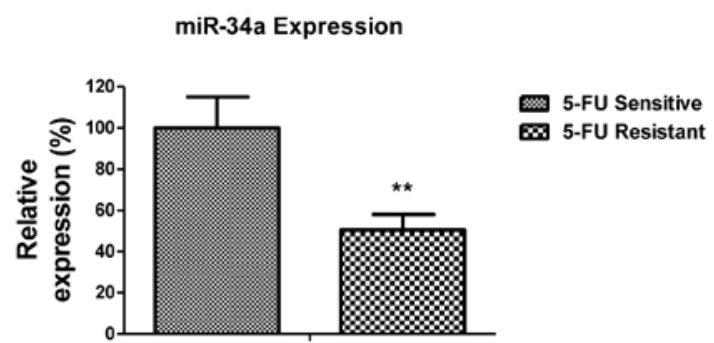

Figure 1. Expression of miR-34a in 5-FU-resistant colon cancer cells. (A) Generation of 5-FU-resistant cells from DLD-1 human colorectal cancer cells. DLD-1 parental cells were treated with gradually increasing concentrations of 5-FU in regular cell culture conditions for selection of resistant cells DLD-1 5-FU-resistant clones were pooled and analyzed by treatment with 5 -FU at the indicated concentrations for $72 \mathrm{~h}$ and cell viability was determined. (B) Expression of miR-3a in a 5-FU resistant cell line compared with a 5-FU sensitive cell line. Columns represent the mean of three independent experiments. Bars represent the standard error. ${ }^{* *} \mathrm{P}<0.05$. 5-FU, 5-fluorouracil; miR-34a, microRNA-34a.

(Dual-Luciferase ${ }^{\circledR}$ Reporter Assay System; Promega; E1910). Luciferase (LUC) activity was measured using a dual luciferase reporter assay (Dual-Luciferase ${ }^{\circledR}$ Reporter Assay System; Promega; E1910). The pRL-TK vector was used as an internal control. The results are expressed as relative LUC activity (firefly LUC/renilla LUC).

Statistical analysis. The data were analyzed using GraphPad 5.0 (GraphPad Software, Inc., La Jolla, CA, USA). Unpaired Student's t-test was used for the data analysis. All data are shown as the mean \pm standard error. $\mathrm{P}<0.05$ was considered to indicate a statistically significant difference.

\section{Results}

miR-34a is downregulated in 5-FU-resistant colon cancer cells. Since miR-34a has been reported to act as a tumor suppressor in a number of tumor types (18) and is associated with 5-FU resistance (17), the present study investigated the role of miR-34a in 5-FU resistance in human colon cancer cells. A 5-FU-resistant cell line was generated using DLD-1 cells, by selection with gradually increasing concentrations of 5-FU in a cell culture medium. Following successive treatments for a duration of three months, several 5-FU-resistant cell clones were developed and pooled for use in the subsequent experiments. To verify resistance, parental cells and resistant pool cells were treated with 5-FU at various concentrations for $72 \mathrm{~h}$. As hypothesized, cell viability assays demonstrated that DLD-1 5-FU-resistant cells tolerated markedly higher concentrations of 5-FU compared with sensitive cells, which exhibited significant inhibition of viability at 4, 20 and $50 \mu \mathrm{M}$ (Fig. 1A). The $\mathrm{IC}_{50}$ was $\sim 5 \mu \mathrm{M}$ for 5-FU-sensitive cells and $45 \mu \mathrm{M}$ for resistant cells. As hypothesized, the expression of miR-34a was significantly downregulated in 5-FU-resistant cells compared with sensitive cells (Fig. 1B), suggesting that miR-34a may act as a tumor suppressor in human colon cancers and that it is involved in the development of resistance to 5-FU.

LDHA is a direct target of miR-34a in colon cancer cells. The initial results showed that miR-34a is downregulated in 5-FU-resistant cells. Potential targets of miR-34a were then investigated. An miRNA database (www.targetscan.org) was searched for predicted targets of miR-125b that may contribute to 5-FU resistance. The public miRNA database,TargetScan, predicted that LDHA may be a target for miR-34a, and showed that the 3'-UTR of LDHA contains a highly conserved binding site for miR-34a (Fig. 2A). To the best of our knowledge, thus far no publication has reported that LDHA is a direct target of miR-34a in colon cancer cells. To determine whether miR-34a targets LDHA in colon cancer cells, pre-miR-34a was transfected into DLD-1 cells. Overexpression of miR-34a significantly downregulated expression of the LDHA protein (Fig. 2B). The following experiment sought to investigate whether miR-34a directly targets the 3'-UTR of LDHA mRNA. A luciferase reporter analysis was performed by co-transfecting a vector containing pMIR reporter-luciferase fused with either the original 3'-UTR sequence or a sequence with a mutation in the predicted binding site of the 3'-UTR of LDHA mRNA, and with either pre-miR-34a or control microRNA. Overexpression of miR-34a decreased the luciferase activity of the reporter containing the wild type 3 '-UTR of LDHA by $\sim 60 \%$ in DLD-1 cells (Fig. 2C). However, no such inhibitory effects of miR-34a on the activity of the reporter fused with the 3'-UTR of LDHA with the mutation in the predicted binding site were detected (Fig. 2C), These results demonstrate that LDHA is a direct target of miR-34a in colon cancer cells.

5-FU-resistant cells exhibit increased expression and activity of LDHA. Previous studies have shown that dysregulated cellular metabolism is associated with 5-FU resistance in cancer cells (19). LDHA catalyzes the final step in the glycolytic pathway, which is the conversion of pyruvate and nicotinamide adenine dinucleotide dehydrogenase to lactate and nicotinamide adenine dinucleotide, and is known to be involved in tumor maintenance. The present study aimed to investigate whether LDHA is involved in miR-34a-mediated 5-FU resistance in colon cancer cells. Notably, the expression of LDHA was upregulated at protein and mRNA levels in 5-FU-resistant cells (Fig. 3A and B), suggesting that the downregulation of miR-34a in 5-FU-resistant cells may contribute to LDHA upregulation. Consistent with this, the activity of LDH and the levels of lactate were increased in 5-FU-resistant cells compared with 5-FU-sensitive cells (Fig. 3C and D). These results demonstrate that LDHA is associated with 5-FU 
Position 406-413 of LDHA3' UTR

hsa-miR-34a

B

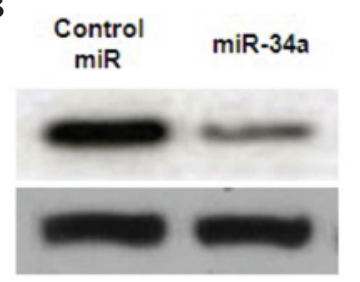

LDHA

$\beta$-actin
5' ...cCAccucugacgcaccacugcca.. ||IIIII

3' UGUUGGUCGAUUCUGUGACGgU

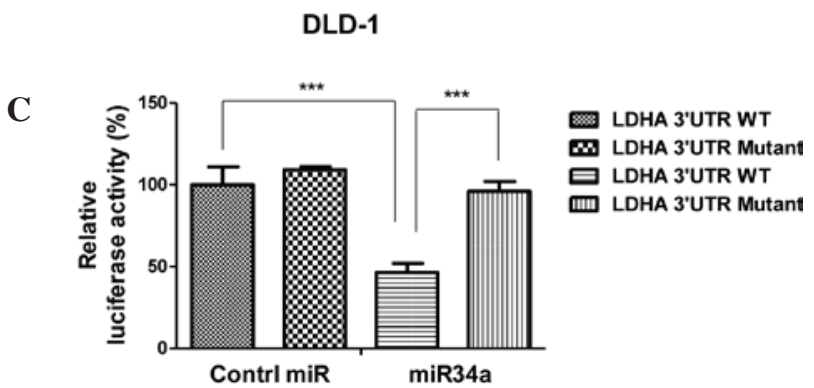

Figure 2. Targeting of LDHA by miR-34a in colon cancer cells. (A) Target prediction from Targetscan.org. The position 406-413 of LDHA 3'-UTR contains putative binding sites for miR-34a. (B) DLD-1 cells were transfected with $100 \mathrm{nM}$ pre-miR-negative (control miR) or pre-miR-34a for $48 \mathrm{~h}$. Cell lysates were prepared for western blotting analysis. $\beta$-actin was used as a loading control. (C) DLD-1 cells were co-transfected with luciferase reporter plasmids with wild type 3'-UTR or mutant 3'-UTR of LDHA and pre-miR-34a, or pre-miR-negative (control miR) using Lipofectamine 2000 reagent. Post-transfection (48 h), cells were harvested and lysed with passive lysis buffer. LUC activity was measured by a dual luciferase reporter assay. The pRL-TK vector was used as an internal control. Results are expressed as relative LUC activity (firefly LUC/ Renilla LUC). Columns represent the mean of three independent experiments. Bars represent the standard error. ${ }^{* *} \mathrm{P}<0.01$, compared with control. LDHA, lactate dehydrogenase A; UTR, untranslated region; miR-34a, microRNA,-34a; LUC, luciferase.

A
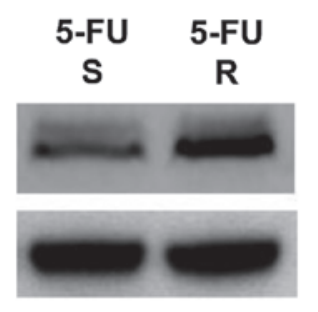

LDHA

$\beta$-actin

LDH Activity

C

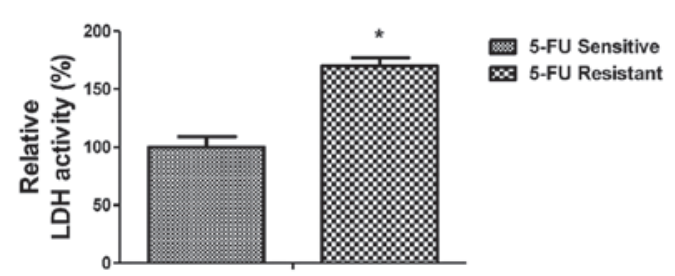

LDHA MRNA

B

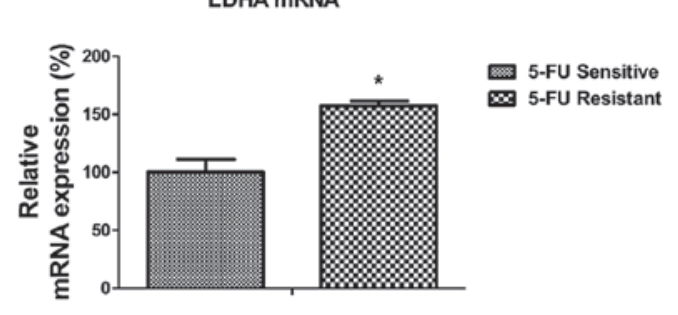

Lactate Product

D

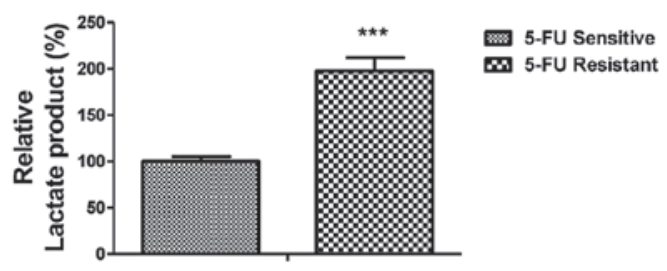

Figure 3. LDHA levels in 5-FU-resistant DLD-1 colon cancer cells. (A) Representative western blot showing the upregulated LDHA protein level in 5-FU-resistant colon cancer cells. $\beta$-actin was used as a loading control. (B) mRNA levels of LDHA was increased in 5-FU-resistant cells, measured by reverse transcription-quantitative polymerase chain reaction. (C) 5-FU-resistant cells displayed higher LDH activity compared with sensitive cells. (D) Lactate product in 5-FU resistant colon cells was elevated compared with sensitive cells measured at $24 \mathrm{~h}$. The relative lactate product was calculated by comparison with control treatment cells. Columns represent the mean of three independent experiments. Bars represent the standard error. ${ }^{*} \mathrm{P}<0.05$ and ${ }^{* * * *} \mathrm{P}<0.001$. LDHA, lactate dehydrogenase A; 5-FU, 5-fluorouracil.

resistance in colon cancer cells and may be a promising therapeutic target.

Overexpression of miR-34a sensitizes 5-FU-resistant cells through direct targeting of LDHA. To investigate the mechanism underlying the association between miR-34a-mediated downregulation of LDHA and 5-FU resistance in colon cancer cells, miR-34a was exogenously overexpressed in DLD-1
5-FU-sensitive and resistant cells through transient transfection. These cells were then treated with increasing concentrations of 5-FU for $72 \mathrm{~h}$. Transfection with miR-34a significantly inhibited cell viability in sensitive and resistant cancer cells (Fig. 4A and B). Compared with control microRNA, overexpression of miR-34a in 5-FU-sensitive cells led to a decrease in $\mathrm{IC}_{50}$ from 4 to $1 \mu \mathrm{M}$. The $\mathrm{IC}_{50}$ of 5-FU-resistant cells in response to 5 -FU decreased from 45 
A

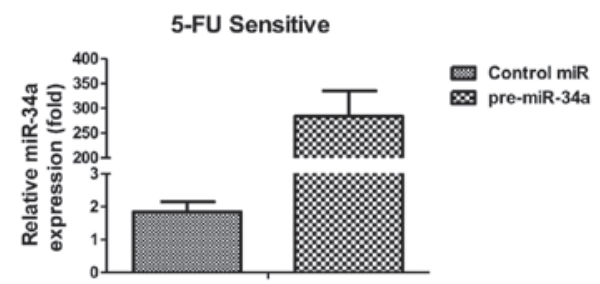

B

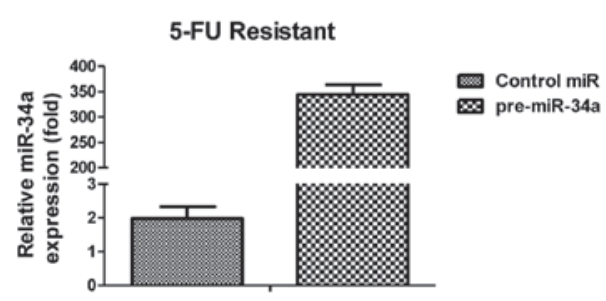

5-FU Sensitive

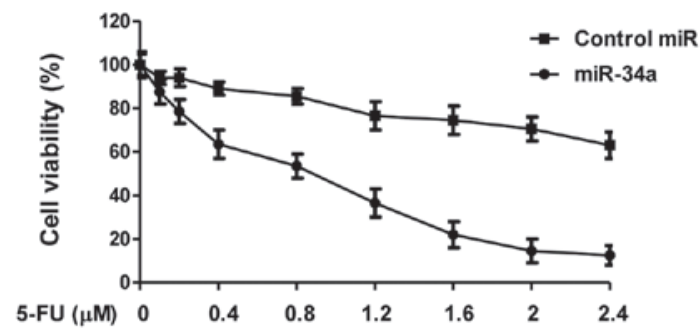

5-FU Resistant

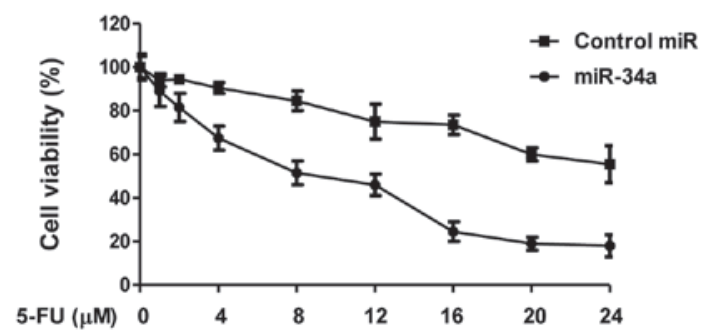

Figure 4. Effect of overexpression of miR-34a on sensitivity of 5-FU-resistant cells to 5-FU. (A) Transient transfection of pre-miR-34a into 5-FU sensitive DLD-1 human colorectal cancer cells (left) for $48 \mathrm{~h}$. Cells were then treated with 5 -FU at the indicated concentrations for 72 h followed by cell viability analysis (right). (B) Transient transfection of pre-miR-34a into 5-FU resistant DLD-1 cells (left) for $48 \mathrm{~h}$. Cells were then treated with 5-FU at the indicated concentrations for $72 \mathrm{~h}$ followed by cell viability analysis (right). Columns represent the mean of three independent experiments. Bars represent the standard error. miR-34a; microRNA-34a; 5-FU, 5-fluorouracil.

A
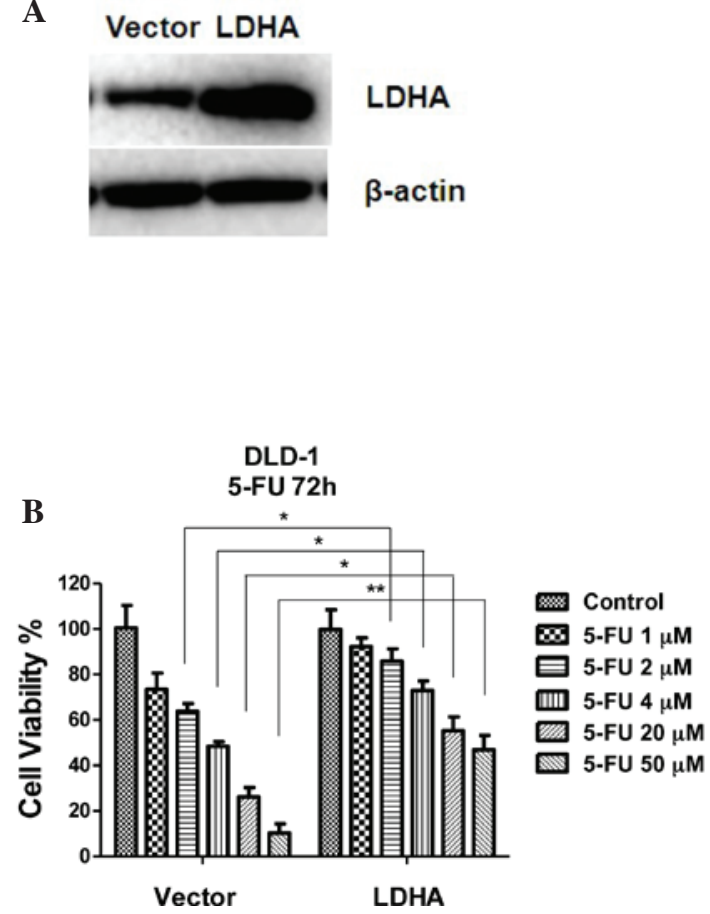

Figure 5. Effect of LDHA levels on colon cancer cell resistance to 5-FU. (A) Expression of LDHA following transient transfection of LDHA into DLD-1 cells. $\beta$-actin was used as a loading control. (B) After transfection (48 h), vector control cells and cells with overexpression of LDHA were treated with 5-FU with indicated concentrations for $72 \mathrm{~h}$ and cell viability assays were performed. Columns represent the mean of three independent experiments. Bars represent the standard error. ${ }^{*} \mathrm{P}<0.05$ and ${ }^{* *} \mathrm{P}<0.01$. LDHA, lactate dehydrogenase A; 5-FU, 5-fluoruracil.

to $8 \mu \mathrm{M}$. To verify whether overexpression of miR-34a sensitizes colon cancer cells to 5-FU treatment through inhibition of LDHA, LDHA was transiently transfected into DLD-1
5-FU-sensitive cells (Fig. 5A) and the sensitivity to increasing concentrations of 5-FU for $72 \mathrm{~h}$ was measured (Fig. 5B). The results demonstrated that exogenous overexpression of LDHA rendered DLD-1 cells resistant to 5-FU, indicating that overexpression of miR-34a results in cells that are susceptive to 5-FU via the inhibition of LDHA.

\section{Discussion}

miRNAs have been shown to be involved in the regulation of a number of processes that are deregulated in cancer cells, such as proliferation, differentiation and apoptosis $(1,2)$. Downregulation of miR-34a has been reported in a number of cancer types, including colorectal cancer (20), pancreatic cancer (21), prostate cancer (22) and neuroblastoma (23). To date, numerous targets of the miR-34 family have been postulated, including mesenchymal-epithelial transition factor (23), cyclin-dependent kinase 6 (24), c-Myc and N-Myc (25), silent mating type information regulation 2 homolog (SIRT1) (26) and Bcl-2 (27). The greatest level of induction by p53 was observed in miR-34a, which has been shown to be a direct target gene of p53 (28). Furthermore, ectopic miR-34 expression induces apoptosis, and cell-cycle arrest or senescence (29). Thus, miR-34a is recognized as a tumor suppressor. A recent study showed that ectopic expression of miR-34a in 5-FU-resistant colon cells inhibited cell growth and attenuated the resistance to 5-FU through the downregulation of SIRT1 and E2F3 (17), suggesting that miR-34a is involved in cancer cell resistance to 5-FU. The results of the present study showed a downregulation of miR-34a expression in 5-FU-resistant colon cancer cells, which suggests a tumor suppressive function of this miRNA in colon cancer cells.

In 1956, Warburg observed that the rate of glycolysis was abnormally high in cancer cells, yet a smaller fraction of this 
glucose is broken down by oxidative phosphorylation. This Warburg effect indicates that the metabolic properties of cancer cells are different from those of normal cells. Cancer cells are more dependent than healthy cells on aerobic glycolysis, fatty acid synthesis and glutaminolysis for proliferation (12). Therefore, targeting cancer cell metabolism may be a selective approach by which to treat cancer patients. Recently, a number of studies have reported that dysregulated metabolism is associated with drug resistance. Cancer cells are known to take up glucose avidly and generate lactate through LDHA, which has been reported to be involved in tumor maintenance and progression. In addition, LDH activity is increased in colon cancer, indicating that LDHA may be of use as a prognostic marker (30). Therefore, as chemoresistant cancer cells exhibit an abnormal metabolism, this could in itself be a target for the development of novel therapeutic agents.

The current study demonstrated that LDHA is a direct target of miR-34a in colon cancer cells. Overexpression of miR-34a decreased LDHA protein levels, which contributed to the re-sensitization of 5-FU-resistant cancer cells. The role of LDHA in acquired 5-FU resistance in human colon cancer cells was investigated. Compared with parental cells, 5-FU-resistant cells exhibited an increase in the expression and activity of LDHA. Overexpression of LDHA resulted in increased resistance to 5-FU. In addition, the current study found that inhibition of LDHA by overexpressing miR-34a significantly re-sensitized 5-FU-resistant cells. This demonstrated the importance of miR-34a-mediated inhibition of LDHA in overcoming chemoresistance in cancer cells. Further investigation into other putative targets of miR-34a is required. This may be facilitated by the use of gene expression profiling approaches to identify signal pathways regulated by miR-34a and their association with sensitivity to 5-FU in order to develop novel therapeutic approaches to overcome 5-FU resistance.

\section{Acknowledgements}

The authors would like to thank the staff and faculty of the Department of Hematology and Oncology, the 101st Hospital of the People's Liberation Army and Dr Haibin Zhao of the Department of Pathology, the 101st Hospital of the People's Liberation Army for his editorial assistance.

\section{References}

1. He L and Hannon GJ: MicroRNAs: small RNAs with a big role in gene regulation. Nat Rev Genet 5: 522-531, 2004.

2. Ameres SL and Zamore PD: Diversifying microRNA sequence and function. Nat Rev Mol Cell Biol 14: 475-488, 2013.

3. Krol J, Loedige I and Filipowicz W: The widespread regulation of microRNA biogenesis, function and decay. Nat Rev Genet 11: 597-610, 2010.

4. Fabbri M, Croce CM and Calin GA: MicroRNAs. Cancer J 14: $1-6,2008$.

5. Slaby O, Bienertova-Vasku J, Svoboda M and Vyzula R: Genetic polymorphisms and microRNAs: new direction in molecular epidemiology of solid cancer. J Cell Mol Med 16: 8-21, 2012.

6. Longley DB, Harkin DP and Johnston PG: 5-fluorouracil: mechanisms of action and clinical strategies. Nat Rev Cancer 3: 330-338, 2003.

7. Curtin NJ, Harris AL and Aherne GW: Mechanism of cell death following thymidylate synthase inhibition: 2'-deoxyuridine-5'-triphosphate accumulation, DNA damage, and growth inhibition following exposure to CB3717 and dipyridamole. Cancer Res 51: 2346-2352, 1991.
8. Peters GJ, van Triest B, Backus HH, Kuiper CM, van der Wilt CL and Pinedo HM: Molecular downstream events and induction of thymidylate synthase in mutant and wild-type p53 colon cancer cell lines after treatment with 5-fluorouracil and the thymidylate synthase inhibitor raltitrexed. Eur J Cancer 36: 916-924, 2000.

9. Giacchettii S, Perpoint B, Zidani R, Le Bail N, Faggiuolo R, Focan C, Chollet P, Llory JF, Letourneau Y, Coudert B, et al: Phase III multicenter randomized trial of oxaliplatin added to chronomodulated fluorouracil-leucovorin as first-line treatment of metastatic colorectal cancer. J Clin Oncol 18: 136-147, 2000.

10. Douillard JY, Cunningham D, Roth AD, Navarro M, James RD, Karasek P, Jandik P, Iveson T, Carmichael J, Alakl M, et al: Irinotecan combined with fluorouracil compared with fluorouracil alone as first-line treatment for metastatic colorectal cancer: a multicentre randomised trial. Lancet 355: 1041-1047, 2000.

11. Vander Heiden MG, Cantley LC and Thompson CB: Understanding the Warburg effect: the metabolic requirements of cell proliferation. Science 324: 1029-1033, 2009.

12. Warburg O: On respiratory impairment in cancer cells. Science 124: 269-270, 1956.

13. Kim JW and Dang CV: Cancer's molecular sweet tooth and the Warburg effect. Cancer Res 66: 8927-8930, 2006.

14. Zhao Y, Butler EB and Tan M: Targeting cellular metabolism to improve cancer therapeutics. Cell Death Dis 7: e532, 2013.

15. Doherty JR and Cleveland JL: Targeting lactate metabolism for cancer therapeutics. J Clin Invest 123: 3685-3692, 2013.

16. Fantin VR, St-Pierre J and Leder P: Attenuation of LDH-A expression uncovers a link between glycolysis, mitochondrial physiology, and tumor maintenance. Cancer Cell 9: 425-434, 2006.

17. Akao Y, Noguchi S, Iio A, Kojima K, Takagi $T$ and Naoe T: Dysregulation of microRNA-34a expression causes drug-resistance to 5-FU in human colon cancer DLD-1 cells. Cancer Lett 300: 197-204, 2011.

18. Hermeking H: The miR-34 family in cancer and apoptosis. Cell Death Differ 17, 193-199, 2010.

19. Zhou Y, Tozzi F, Chen J, Fan F, Xia L, Wang J, et al: Intracellular ATP levels are a pivotal determinant of chemoresistance in colon cancer cells. Cancer Res 72: 304-314, 2012.

20. Akao Y,NakagawaI,HirataI,Iio A,ItohT,KojimaK, NakashimaR, Kitade Y and Naoe T: Role of anti-oncomirs miR-143 and -145 in human colorectal tumors. Cancer Gene Ther 17: 398-408, 2010.

21. Chang TC, Wentzel EA, Kent OA, Ramachandran K, Mullendore M, Lee KH, Feldmann G, Yamakuchi M, Ferlito M, Lowenstein CJ, et al: Transactivation of miR-34a by p53 broadly influences gene expression and promotes apoptosis. Mol Cell 26: 745-752, 2007.

22. Rokhlin OW, Scheinker VS, Taghiyev AF, Bumcrot D, Glover RA and Cohen MB: MicroRNA-34 mediates AR-dependent p53-induced apoptosis in prostate cancer. Cancer Biol Ther 7: 1288-1296, 2008.

23. Yan D, Zhou X, Chen X, Hu DN, Dong XD, Wang J, et al: MicroRNA-34a inhibits uveal melanoma cell proliferation and migration through downregulation of c-Met. Invest Ophthalmol Vis Sci 50: 1559-1565, 2009.

24. Sun F, Fu H, Liu Q, Tie Y, Zhu J, Xing R, et al: Downregulation of CCND1 and CDK6 by miR-34a induces cell cycle arrest. FEBS Lett 582: 1564-1568, 2008.

25. Wei JS, Song YK, Durinck S, Chen QR, Cheuk AT, Tsang P, et al: The MYCN oncogene is a direct target of miR-34a. Oncogene 27: 5204-5213, 2008.

26. Yamakuchi M, Ferlito M and Lowenstein CJ: miR-34a repression of SIRT1 regulates apoptosis. Proc Natl Acad Sci USA 105: 13421-13426, 2008.

27. Yang F, Li QJ, Gong ZB, Zhou L, You N, Wang S, Li XL, Li JJ, An JZ, Wang DS, et al: MicroRNA-34a targets Bcl-2 and sensitizes human hepatocellular carcinoma cells to sorafenib treatment. Technol Cancer Res Treat 13: 77-86, 2014.

28. Ji Q, Hao X, Meng Y, Zhang M, Desano J, Fan D and Xu L: Restoration of tumor suppressor miR-34 inhibits human p53-mutant gastric cancer tumorspheres. BMC Cancer 8: 266, 2008.

29. Welch C, Chen Y and Stallings RL: MicroRNA-34a functions as a potential tumor suppressor by inducing apoptosis in neuroblastoma cells. Oncogene 26: 5017-5022, 2007.

30. Koukourakis I, Giatromanolaki A and Sivridis E (eds): Colorectal cancer: Lactate dehydrogenase (LDH) activity as a prognostic marker. In: Methods of Cancer Diagnosis, Therapy, and Prognosis, Springer, Berlin, Germany, Vol 4, pp241-253, 2009. 